\title{
Turning Users' In-Game Behaviours into Actionable Adaptive Gamification Strategies using the PEAS Framework
}

\author{
Enrica Loria \\ Graz University of Technology \\ Graz, Austria \\ eloria@tugraz.at
}

Lennart E. Nacke

Stratford School of Interaction Design and Business, University of Waterloo, Canada lennart.nacke@acm.org

\begin{abstract}
Adaptive gamification answers the need to customize engagement strategies because users are motivated by different game elements and mechanics. To better understand these individual preferences, user modelling is vital. However, gameful designers must make many decisions on matching profiling data to actual adaptation strategies, which makes modelling particularly challenging. The lack of a standardized and guided process for adaptive gamification hinders replicability, comparability, and complicates making adaptation dynamic. In this study, we analyzed a persuasive gameful application (Play\&Go) to show how in-game behaviours can be translated into adaptation strategies. We used an existing adaptation framework (PEAS) grounded in the games and gamification literature. Our work demonstrates the suitability of the PEAS model as a shared, standardized method for adaptive gamification and shows how it can guide the process of transforming user behaviours into actionable adaptation strategies.
\end{abstract}

\section{Introduction}

Gamification research has demonstrated the need for adaptation to account for interpersonal differences in the perception of gameful design elements [?, 1, 2]. Adaptation means tailoring the game elements or mechanics for each user, using models that describe the user's traits and behaviours within the gameful application. Such properties may include user behaviour, playstyle, and motives [3, 4]. Researchers demand more adaptive content in gameful environments, including more elaborate profiling techniques, as well as embedding cyclical (or dynamic) tailoring. Recent studies (e.g., [5, 6, 7])

\author{
Maximilian Altmeyer \\ German Research Center for \\ Artificial Intelligence (DFKI), Germany \\ maximilian.altmeyer@dfki.de
}


for green mobility (i.e., Play\&Go). Additionally, we discuss the advantages of using such an approach for both researchers and practitioners, and how this method can help answer open research problems in adaptive gamification. Therefore, by answering this question, we are making the following contributions to the gamification community: (1) a method to derive the adaptation strategy (from the PEAS framework), (2) a method to operationalize users' profiles into adaptation strategies using the PEAS framework, and (3) a use case showing the advantages of using this approach for dynamic adaptation, replicability, and comparability.

\section{Background and Related Works}

\subsection{Gamification Panorama}

In its simplest, most common definition, gamification is described as the use of game elements in non-game contexts [13]. It has been used under this term for about a decade in educational settings to increase learner performance, motivation, or engagement [14], and to make users more intrinsically motivated in performing a certain task [15]. Previous studies have shown that gamification can turn unpleasant tasks into fun ones and enhance the user experience in numerous contexts and domains [16]. In addition to academia, gamification has leaped the industry and has become an established practice in user experience design [17], while also gaining popularity in different domains [18]. However, gamification is not always successful, as its effects highly depend on its context and implementation [16]. Hence, research has focused on improving the design of such systems to maximize their success rate [19], e.g., engagement and the pursuit of the underlying goal [18].

\subsection{Adaptive Gamification}

Implementing gameful mechanics does not implicate automatic increases in users' activity [20]. Designing gameful applications is challenging as playing should be a voluntary, intrinsically motivated behaviour [21]. However, gameful applications generally promote actions users tend to avoid. To achieve intrinsic motivation, a behaviour has to fulfill the needs for competence, autonomy, and relatedness [22]. Nevertheless, the satisfaction of such motivational affordances is highly personal [22]. It derives that treating users as a homogeneous unit is an unsuccessful strategy [23]. Thus, the gameful affordances implemented in gamification research should be diversified to allow the creation of more inclusive gameful experiences [18].
Many recent studies have embraced this research trend and investigated ways to transform gameful applications into adaptive experiences. Researchers demonstrated the need for more dynamic tailoring, also relying on other features than static user profiles [24]. Therefore, the usage of implicit data, as telemetry logs, is encouraged. Within games research, user profiling and content generation already heavily rely on data-driven approaches (e.g., $[25,26]$ ), confirming how content adaptation can lead to higher motivation and longer-term engagement [7]. This trend is also picking up in gamification research. For instance, in gamification for education, researchers show how adapting gaming features led learners to spend significantly more time in the learning environment, as well as showed they were more motivated than students engaging with static gameplay [6]. In those examples, generally, the adaptation is guided by profiles build using user types taxonomies (e.g., the Hexad User Types [27]). Yet, recently, other approaches have been investigated, combining profiles from different sources [28] (e.g. dominant user type, personality or gender), which was found beneficial in improving intrinsic motivation and decreasing amotivation. In other cases, when researchers use users' data, they cluster users' behaviours to distinguish among engaged and unengaged users and identify user types [29] or other Machine Learning techniques to discover users' preferences from telemetry data [30]. Conversely to most studies claiming the benefits of tailored gamification, less successful examples also exist [31]. However, findings of this kind, rather than suggesting the inutility of adaptation, are additional proof that adapting the gaming experience is a challenging task.

Nevertheless, those studies are linked by a common denominator: they follow a very context-specific approach, without relying on an adaptation framework. In the gamification literature, few adaptation frameworks exist. These generally should be used at the design level. For example, Bockle et al. [8] proposed a framework to inform the systematic development of adaptive gamification systems by providing design path and principles, leading to a requirements' template. Loria [10], on the other hand, presented a conceptual framework for dynamic tailoring, which structures the adaptation phases in a cycle, while still remaining at a high level. Finally, Knutas et al. [9] delineated a process to include personalization blocks in the design of gamified applications. Yet, it still lacks a framework to translate in-game activity or user profiles into actionable adaptation strategies that can be highly beneficial. Not only would it guide designers in the ideation of their adaptation strategies while considering the specific 
domain, but it would also help to produce solutions that are easier to interpret, replicable (which is a known issue [32]), and comparable.

All in all, the field of adaptive gamification is still young and growing, especially for what concerns dynamic adaptation [5]. As such, gamification research still misses coherence in research models, as well as theoretical foundations [18]. Yet, the conversation is still focused on what can and needs to be personalized, rather than on how to tailor experiences [24]. Scholars and practitioners should be guided in how to utilize implicit behavioural data to update and improve the gamification model in a cyclic manner [18, 24], benefiting by periodically producing novel content [18]. A standard approach to convert users' (dynamic) profiles into actionable adaptation strategies could contribute to the quest of producing adaptive systems. Yet, it is still missing, to our knowledge.

\subsection{The PEAS Framework}

The previously mentioned frameworks were lacking a concrete, guided procedure to generate adaptation strategies agnostic to the application domain. This lack of generalizability depends on the frameworks treating the problem at a very high level. Therefore, implementing the frameworks comes with a series of choices dictated by the specific domain. Consequently, replicability and comparability are difficult to achieve. Conversely, the literature offers a design framework informed by a review on both games and gamification research: the PEAS framework [11]. The PEAS framework describes the various axes on which designers can adapt their system in a hierarchical manner. It is divided into four macro components: player, environment, agents, system. Player describes every facet of the player and their character, including their appearance and the actions allowed. Environment describes both the aesthetic and narratological aspects of the virtual world. Agent describes the game characters not controlled by the players, e.g., enemies or companions. System describes functional aspects of the game, as the game dynamics and rules. Each of these macro components is composed of a set of mid-level-components that are themselves composed of micro-level components that designers can adapt. For example, the Player component is composed of Representation and Action Space. If a designer wishes to adapt the players' representation, they can make a change on their characteristics or personality for example. A complete overview of the PEAS components is presented later (Table 2). The authors also provided a list of guiding questions to assist the framework's instantiation in specific applications i.e assigning various context specific elements to the four macro components.

The PEAS framework has been extended [12] in a generalized model for player profiling to obtain a homogeneous representation of players' preferences over different game elements. Thus, this generalized model allows combining player and personality approaches to produce a single adaptation strategy, assuming that there are no conflicts among the representation. The model is structured in phases, one of them being the model translation. While blending different player profiling methods is out of the paper's scope, the translation function is indeed relevant. Specifically, they provided a translation for players' behaviours into the PEAS representation, in the form of a numerical vector.

In summary, the PEAS framework comes with a series of advantages. First, it allows the definition of a wide variety of adaptation aspects, resulting from a rigorous systematic review. Second, its hierarchical structure allows decoupling the adaptation axes and separately analyzing them. Finally, the framework is further supported by a peer-reviewed translation method, which assists our needs for a validated proxy and methodology to use it.

\section{Method and Materials}

In the following section, we will introduce our use case: the gameful app Play\&Go, as well as the in-game behaviours extracted from telemetry data and defined with the support of the games' designers and domain experts. Play\&Go data is relevant to our research as (1) the system meets the description of gamification, as game-like elements are used to foster an ulterior goal: greener transport habits; (2) the system is in continuous development, so research outcomes can be used to improve users' experience further; (3) the app is publicly available and free to download, and thus, the only selection criteria for participants was for them playing within a limited geographic area; and (4) the designers shared the anonymized data, upon request, and were available for interviews aimed at a deeper understanding of the gameful environment.

\subsection{Play\&Go}

We analyzed data from the users' interaction in Play\&Go [33], a persuasive gamified application with the goal of promoting ecological transportation behaviours among the citizens active in the region of Trentino (Italy). Users can track their movements within the region by selecting a transportation means 
among the ones supported (walk, bike, bus and train) and see their current and past activity in their profile. An algorithm validates tracked trips, which, if valid, award Green Leaves points according to the trip's length and level of sustainability (e.g., bike > bus). Users' can monitor the leaderboard to check their position in contrast to other users in both a global and weekly leaderboard. The former encourages users to continue to play the game over the 6-month campaign, while the latter ensures fairness for newcomers. Users can search other users in the leaderboard and visit their profiles to visualize their achievements and activity. On the homepage, users can view their current level (Green Lover) and how many points (Green Leaves) are needed to reach the following level. The levelling system is associated with an unlocking mechanism. As users progress in the game, they can have new or upgraded features. The homepage allowed users to see an overview of the challenges currently active and their progress towards completing them. Each challenge is valid for one week. Challenges are a core mechanic in the gamified app and can either be single-player or multiplayer. For newcomers, single-player challenges are automatically assigned. When users reach level 2, they can choose one of two single-player challenges each week. Reaching level 3 increases the pool of challenges to three. Starting from level 4 , users can participate in 2-player challenges, either competitive or cooperative. To activate multiplayer challenges, users can either send an invite to another user or accept a received invitation.

Dataset. For the analyses presented in this paper, data has been collected from November the 2nd, 2019 to February the 28th, 2020, for a total of 17 weeks. Citizens join the campaign voluntarily and, upon registration, they are required to fill out a questionnaire gathering information on age/gender and daily transportation habits. Users were very well-distributed among males and females, both representing half of the population, with a variation of few percentile points. Users' age distribution was also consistent throughout the years. Two-thirds of the users were in the age range 20-35 and 35-50 years old, one-third for each category. Younger (16-20 years old) and older (50-70 years old) users were less present, amounting to one-fifth of the population each, on average. A very small minority of the users were older than 70 years. In the dataset, we count 425 registered users, of whom about 127 of them where considered for the analysis. The filtering choice was dictated by the need of having users above Level 3 to compute all the in-game behaviours listed below.

\subsection{User Behaviours}

For Play\&Go, we tracked all of users' game actions, such as trips tracked, levels obtained, interactions with the invitation system, and information on single-player and multiplayer challenges. In collaboration with Play\&Go designers, we defined a list of relevant behaviours for the environment examined, describing users' activity from different perspectives and different granularity levels. The brainstorming session was conducted using a bottom-up and top-down approach. The designers were asked to write down as many in-game behaviours they could think of stating from the list of tracked features. They then wrote another list considering the core aspects of the app: customization, level of activity, social aspect, gamification goal, and will to improve. From the whole joint list replicated concepts were removed and a final set of behaviours were drawn (summarized in Table 1).

Reactivity (R) measures users' speed in answering to game events (e.g., choice of single-player challenges and reply to invitations). Gameplay is divided in weeks, and each week users have 4 days (Tuesday to Friday) to select single-player or multiplayer challenges. Reactivity for a week is measured as the percentage of the 4 days spent before making a choice, if any. The Reactivity value is the average of all the weekly Reactivity for active weeks. A week (or a day) is active if the user performed at least one game action within that week (or day).

Sociality (S) measures users willingness to play with others, either in competitive or cooperative multiplayer challenges. This is manifested through in-game invites to multiplayer challenges. Hence, for this behaviour we measured the number of invites sent by the users. Measuring the number of invites is different than measuring the number of multiplayer challenges, as users may want to play with somebody, hence sending the invites, but may have no invites accepted, and therefore cannot complete the challenges.

Intensive Usage (IU) measures how important users' in-game activity is. The more game actions they perform the more points they get, and thus the sooner they level up. In practice, Intensive Usage is the average of the time spent to reach a new level, weighted for the maximum level reached to avoid penalizing higher (harder to reach) levels.

Winning Social (W) measures whether users tend to win more in multiplayer rather than single-player challenges. This tendency is measured as users' win ratio in multiplayer challenges over their win ratio in single-player challenges.

Full-usage (F) measures the percentage of features 
Table 1: Original list of the behaviours defined in the brainstorming session with the designers and domain experts.

\begin{tabular}{cll}
\hline ID & \multicolumn{1}{c}{ Name } & \multicolumn{1}{c}{ Description } \\
\hline$R$ & Reactivity & Describes users' velocity in answering to game events. \\
$S$ & Sociality & Describes users' tendency to be initiators of multiplayer challenges. \\
$I U$ & Intensive Usage & Describes users' velocity in levelling up. \\
$W$ & Winning Social & Describes users winning rate in multiplayer challenges in contrast to single-player challenges. \\
$F$ & Full-usage & Describes the percentage of features used. \\
$C m$ & Competitive & Describes users' preference of competitive over cooperative multiplayer challenges. \\
$A$ & Active & Describe users' tendency to choose rather than having automatically assigned single-player \\
& & and multi-player challenges. \\
$C s$ & Constancy & Describes the percentage of active days throughout the gameplay. \\
$P o$ & Purpose-oriented & Describes the ratio of green (walk/bike) trips and kms over the trips and kms tracked. \\
$S t$ & Striving & Describes the tendency to engage in difficult challenges. \\
$S i$ & Self-improvement & Describes the tendency to increase and improve the personal performance over the gameplay. \\
\hline
\end{tabular}

used by the user. The complete set of the features available is: each transportation means (walk, bike, bus, and train), the invitation system (send/accept invites), customization of single-player challenge, the unlocking mechanism for multiplayer challenges. Some features are made available to users as they advance in the app. Thus, for each user the Full-usage behaviour is evaluated upon the features available to them.

Competitive $(\mathrm{Cm})$ measures users preference towards competitive challenges. The value is obtained as the ratio of invites to competitive challenges over the cooperative challenges, both sent and accepted.

Active (A) measures users' inclination towards customization. In practice, this value is computed as the ratio of customized challenges (e.g., chosen single-player challenges and multiplayer challenges derived from invites) over challenges automatically assigned by the system - i.e., how active a user was in their tailored gamification experience.

Constancy (Cs) measures whether users were constant in their participation rather than having peaks of activity and many non-active days. Constancy is therefore the percentage of active days within users' gameplay (from their registration day to the last active day).

Purpose-oriented (Po) measures how ecological users transportation behaviours are, considering the trips tracked. Purpose-oriented behaviour evaluates how in line users are with the gamification's purpose: sustainable mobility. It is computed as the number of green (walk and bike) trips over the trips tracked.

Striving (St) measures users will to challenge themselves through difficult tasks. Challenges have associated to them a difficulty value computed according to the challenge's target and the users' history and skills. Striving is computed as the ratio of difficult challenges users' choose.

Self-improvement $(\mathrm{Si})$ measures whether users improved their performance over time, in terms of green kilometres tracked. The self-improvement value represents the slope of the plot of users' activity. Higher the value the more drastic the improvement.

Conceptually, the behaviours present some overlap. For instance, the Active behaviour models users' tendency to actively customize their challenges, while the Reactivity behaviour models users' velocity to reply to app events. Although these events are mostly customization events for challenges, the velocity of users' reaction to them is evaluated. On the other hand, if we find that users either respond very quickly to those events or do not reply at all, Reactivity and Active behaviours become very similar. Thus, before translating these behaviours into the PEAs framework we performed Principal Component Analysis (PCA) [34] to ensure data independence and remove redundant information (see section 4.1).

It should be noted that the behaviours do not evaluate behavioural change - i.e., shift from a least to a more sustainable transportation means. Such information cannot be inferred as users can track a subset of their actual trips and could omit some non-green movements. On the other hand, we can measure a usage increase. Despite an increase in green mobility not implying fewer kilometres with other means, getting users used to move by walk or bike may result in preferring them whenever possible.

\subsection{Using the PEAS Framework in Play\&Go}

In the PEAS framework, the model's definition is guided by the elements that can be personalized within the specific application domain. In Play\&Go, challenges represent the game element that can be adapted towards the users. We will analyze different challenge adaptation strategies for users, derived from 
Table 2: PEAS framework hierarchical structure.

\begin{tabular}{|c|c|c|c|}
\hline \multicolumn{2}{|c|}{ Player } & \multicolumn{2}{|c|}{ Agents } \\
\hline Representation & $\bar{A}$ Action Space & Adversarial & Non-Adversarial \\
\hline $\begin{array}{c}\text { Characteristics } \\
\text { Personality }\end{array}$ & $\begin{array}{l}\text { Skills/Stats } \\
\text { Controls }\end{array}$ & $\begin{array}{c}\text { Individual } \\
\text { Managerial }\end{array}$ & $\begin{array}{c}\text { Companion } \\
\text { Non-companion }\end{array}$ \\
\hline \multicolumn{2}{|c|}{ Environment } & \multicolumn{2}{|c|}{ System } \\
\hline Physical & Narratological & Goals & Rules \\
\hline $\begin{array}{c}\text { Layout } \\
\text { Appearance }\end{array}$ & $\begin{array}{l}\text { Structure } \\
\text { Content }\end{array}$ & $\begin{array}{l}\text { Explicit } \\
\text { Implicit }\end{array}$ & \\
\hline
\end{tabular}

their in-game behaviours.

For the definition of the PEAS representation, the authors define a list of guiding questions [11] revolving around the game element(s) that can be modified.

GQ1. Why are you personalizing your game system?

GQ2. Why did you choose to personalize these game aspects?

GQ3. How will the chosen aspects be personalized?

GQ4. What game aspects have you chosen to personalize? How did you personalize?

We decided to personalize users' experiences to meet their preferences (GQ1). The game aspect chosen for the adaptation are challenges, as is the only game element complex enough to be modified and adapted (GQ2). The adaptation strategy will be computed from users' in-game behaviours (GQ3). Finally, we defined the aspect that can be tailored in the challenges by choosing among the elements available in the hierarchical structure of the framework (Table 2). The dimensions of the PEAS representation are:

Player-Control (PC) refers to users' action space and the control they have over the experience. In Play\&Go, Player-Control can be associated with users' control over challenge assignment mode. Challenges can either be chosen by users from a pool of options or can be automatically assigned, in case an explicit choice was not performed. Thus, this adaptation aspect governs the possibility of customizing (player's choice) or personalizing (system's choice).

System-Rules-Difficulty (SRD) refers to specific rules of the system. The System-Rules sub-component can be further specified in each application domain. With System-Rules-Difficulty, we model the desired difficulty level for the challenge, which can either be easy or hard. System-Rules-Social (SRS) refers to the social aspect of challenges, which can either be present or not. This sub-component governs the challenge and whether it is single-player or multiplayer.

System-Rules-Competition (SRC) refers to the social mechanic used for multiplayer challenges: cooperative or competitive. This dimension is relevant only if the
Table 3: Principal Component Analysis (structure matrix) for 11 user behaviours in Play\&Go $(\mathrm{N}=127)$. For improved visualization, the loading $<.4$ (absolute values) are suppressed. The elements in bold represent the behaviours kept.

\begin{tabular}{lccccc}
\hline Behav. & PC1 & PC2 & PC3 & PC4 & PC5 \\
\hline$R$ & $\mathbf{- 0 . 6 2 8}$ & & & & \\
$S$ & & & & $\mathbf{0 . 8 2 6}$ & \\
$I U$ & & & & & 0.488 \\
$W$ & & & & & \\
$F$ & & & & & \\
$C m$ & & & $\mathbf{0 . 8 7 0}$ & & \\
$A$ & 0.416 & & & & \\
$P p$ & & $\mathbf{0 . 9 7 8}$ & & & \\
$S t$ & & & & & \\
$S i$ & & & & & $\mathbf{0 . 6 9 1}$ \\
$C s$ & & & & & \\
\hline \% Variance & 47.330 & 16.099 & 10.497 & 6.659 & 5.144 \\
\hline
\end{tabular}

System-Rules-Social decides for the challenge to be multiplayer.

System-Rules-Green (SRG) refers to the target of the challenge. Specifically, this sub-components decides whether the challenge will be focused on green transportation means (walk and bike).

Please note that in the original paper [12], the authors represented users on four axes, corresponding to the model's components. In this study, we decided to keep the element of the System macro-component decoupled. They refer to very diverse concepts in Play\&Go and condition different aspects of the adaptation strategy. In other words, each element tackles a distinct challenge setting (e.g., difficulty or sociality) and, as such, they need to be treated separately.

\section{Analyses and Results}

In the following section, we, first, present a preliminary analysis to remove redundancy across our features and, then, we show how the PEAS framework can be used to tailor Play\&Go gameplay.

\subsection{Preliminary Analysis}

We, first, conducted a PCA to remove redundancy in the user behaviours defined. Prior PCA, we verified the sample size adequacy. Empirical rules suggest having 10-15 participants per variable [34]. In our case, the variables are user behaviours (\#11). Hence, we have $\sim 13$ participants $(N=127)$ per variable. Besides, the Kaiser-Meyer-Olkin (KMO) measure of sampling adequacy was .81 , meaning that the sample was large 
Table 4: Translation function for user behaviours to the PEAS representation.

$f_{\text {behav }}(R, S, C m, P o, S i)=$
\begin{tabular}{ccccc}
\hline PC & SRD & SRS & SRC & SRG \\
\hline $\mathrm{R}$ & $\mathrm{Si}$ & $\mathrm{S}+\mathrm{Cm}$ & $\mathrm{Cm}$ & $\mathrm{Po}$ \\
\hline
\end{tabular}

enough to perform the analysis, and Bartlett's Test of Sphericity was significant $\left(\chi_{55}^{2}=612.38, p<.001\right)$, indicating that the correlations between the variables were large enough. In conclusion, our sample of $N=$ 127 was adequate. The analysis was computed using the Python sklearn package. We employed an Oblimin rotation because we expected that the components could partially overlap. An inspection of the scree plot showed a large drop in the eigenvalues after the fifth component. Hence, we retained five components.

Having conducted the PCA, we considered factor loadings $>.512$ (in absolute value) as significant, as suggested in [34] for a sample size of $\sim 100$ and $\alpha=.01$. As Table 3 shows, each of the component has a loading greater than $>.512$ for only one behaviour: PC1, PC2, PC3, PC4, and PC5 represent the Reactivity (R), Purpose-oriented (Po), Competitive (Cm), Sociality (S), and Self-improvement (S) behaviours, respectively. Those behaviours were uncorrelated among one another. In conclusion, among the 11 user behaviours, we retained: Reactivity, Sociality, Competitive, Purpose-oriented, and Self-improvement, which we then translated into the PEAS representation.

\subsection{Translations to the PEAS Framework}

Users' preferences were modelled through in-game behaviours, as previously defined. The mapping to the PEAS elements was conducted in counselling with the designers following a similar approach as in the definition of the in-game behaviours. In addition, designers were asked to engage in a conversation to justify their translations and to agree on a single, final version. In this final formula, Player-Control is positively related to Reactivity, as we can assume that users performing (quick) choices are interested in customizing their game element. System-Rules-Difficulty is positively related to Self-improvement, as the will of improving the own performance can be translated into increasingly difficult tasks. System-Rules-Social is positively related to Sociality and Competitive, as the first behaviour highlights users' will to play with others and the second, in Play\&Go, relates to (competitive) multiplayer challenges. System-Rules-Competitive is positively related to Competitive, for the coherence of the definitions. System-Rules-Green is positively related to Purpose-oriented, as the gamification goal of the app (purpose) is assuming green transportation behaviours. Table 4 summarized the translation of user behaviours into the PEAS representation. Each axes of the PEAS representation (i.e., PC, SRD, SRS, SRC, and SRG) is mapped to one or more users' behaviours. When multiple behaviours are related to one axis, we computed a weighted sum to maintain each score in the range $[0 ; 1]$ (as in [12]).

\subsection{Evolution of the Adaptation Strategies}

In the following section, we emphasize further how having a structured and standard approach for tailoring can benefit the important requirement of cyclic (or dynamic) adaptation. Towards this, we show how easily change in behaviours and in the related adaptation strategies can be modelled. In Play\&Go, each strategy is a numeric vector of dimension 5 (i.e., PC, SRS, SRC, SRD, and SRG), where each element is a value normalized in the range $[0 ; 1]$. As a naive approach, we considered a binary decision where we set a threshold of 0.5. For each element of the vector, when the value is higher than the threshold, the corresponding aspect is embedded in the adaptation strategy: e.g. if $\mathrm{SRS}=0.7$, then the challenge will be multiplayer. We have 32 potential combinations of those aspects - i.e. 32 potential adaptation strategies $\left(2^{5}\right.$, where 2 are the possible choices and 5 the aspect of the PEAS framework we implemented). Each of these adaptation strategies therefore represents a set of challenges proposed to users. For example \#12 represented difficult multiplayer challenges (SRS and SRD active) whereas \#13 represented difficult competitive challenges (SRS, SRD, and SRC active). For illustrative purposes, we divided our observation period into four months (each made of 4 gameplay weeks) and analyzed how users' adaptation strategies changed over time. This investigation shows that as in-game behaviours change over time, the related adaptation strategies are likely to change as well. Figure 1 shows how the adaptation strategies vary in time. Table 5 shows the different possible adaptation strategies, as well as an example of the challenges provided for each one. For example, some of the users for which we would initally select strategy \#22, would be associated to the strategies \#14 or \#6 during the second snapshot. Then, in the third snapshot, for almost half of them, strategy \#18 would be better. In conclusion, this suggests that behaviours, and relative preferences, are likely to change throughout 
Table 5: Overview of the different adaptation strategies. Active dimensions are represented with a "+", un-active with a "-". Strategies 1,3,5,7,17,19,21 and 23 are not represented as they are not viable (competition is active but sociality is not).

\begin{tabular}{cccccc}
\hline Strategy n $^{\circ}$ & PC & SRS & SRD & SRG & SRC \\
\hline 0 & - & - & - & - & - \\
2 & - & - & - & + & - \\
4 & - & - & + & - & - \\
6 & - & - & + & + & - \\
8 & - & + & - & - & - \\
9 & - & + & - & - & + \\
10 & - & + & - & + & - \\
11 & - & + & - & + & + \\
12 & - & + & + & - & - \\
13 & - & + & + & - & + \\
14 & - & + & + & + & - \\
15 & - & + & + & + & + \\
16 & + & - & - & - & - \\
18 & + & - & - & + & - \\
20 & + & - & + & - & - \\
22 & + & - & + & + & - \\
24 & + & + & - & - & - \\
25 & + & + & - & - & + \\
26 & + & + & - & + & - \\
27 & + & + & - & + & + \\
28 & + & + & + & - & - \\
29 & + & + & + & - & + \\
30 & + & + & + & + & - \\
31 & + & + & + & + & + \\
\hline & & & & &
\end{tabular}

time. By modelling the adaptation strategy through the PEAS framework, we can clearly represent and accommodate these changes.

\section{Discussion}

User modelling is often finalized at the deployment of adaptive game content. However, translating those profiles into actionable adaptation strategies might not be straightforward. Towards this, we showed how the PEAS framework, derived from a systematic literature review of games and gamification studies, can indeed be employed for tailoring gameful applications. We then provided a use case example to illustrate the advantages that using this approach can bring to the gamification community, as well as its potential in fulfilling recent needs for dynamic [24, 18] and multi-profiling tailoring [5]. First, the PEAS hierarchical structure combined with the four guiding questions can help disentangle the complexity of

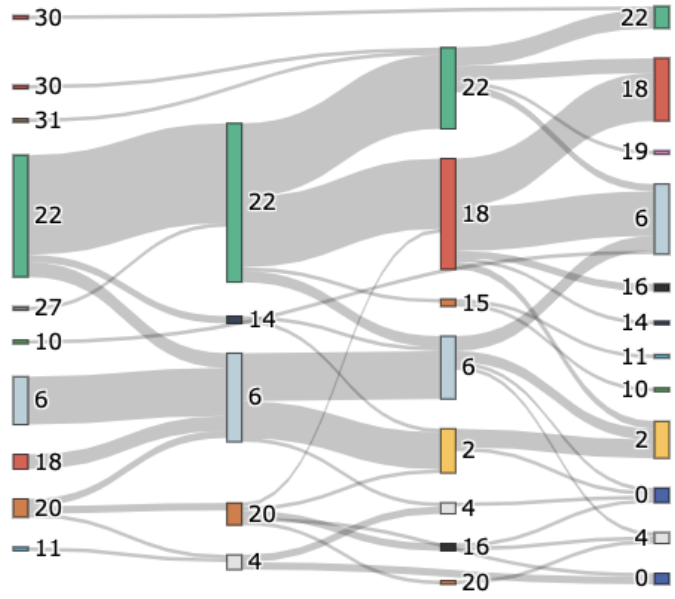

Figure 1: Sankey diagram showing how the adaptation strategies extracted from users' behaviours can change throughout gameplay.

defining the elements to tailor, and how this tailoring can be achieved. Additionally, the structure allows identifying non-customizable aspects, which might limit the interested audience. For instance, in Play\&Go, the Environment component is entirely absent, and thus, users motivated by immersion and aesthetics could be less attracted by the application. Hence, designers might decide to include game elements to embrace a broader users base. Second, this approach embraces the concept of trait-based profiling and adaptation, which is more accurate and versatile than approaches based on user types [35]. Third, this approach fosters replicability in a similar domain, even though the low-level definition of users' behaviours may differ. Consequently, not only can works be compared more easily, but researchers can also exploit this method to test and evaluate how different modelling techniques lead to different adaptation strategies and, then, make a more conscious decision on the approach to be implemented. Fourth, using the PEAS framework can assist the deployment and update of dynamic adaptation. In this regard, we provided an example of how profiles built from telemetry data can change and impact the related adaptation strategy, regardless of the simplicity of the game mechanics. In Play\&Go, we had both evidence of users having a stationary and changeable behaviour in terms of in-game activity. Moreover, using the PEAS framework allowed to clearly display when and how variation in in-game activity implied a change in the adaptation strategy. Finally, having a structured adaptation strategy enables multi-profile adaptation. Although we did not present a concrete example on 
how to combine different profiling techniques, this is detailed by the authors of the PEAS framework [12]. It is sufficient to define a mapping for each type of model and combine the resulting vectors with the PEAS translations. The final vector will be used to implement the adaptation strategy. In conclusion, we advise researchers and practitioners to employ the PEAS adaptation framework to foster replicability and comparability of approaches and findings, for either static or dynamic tailoring of gameful experience.

\subsection{Implications for Adaptive Gamification}

Adaptive gamification is a timely topic, gaining interest from both researchers and practitioners. Consequently, our work has implications in both academia and in the industry. First, using the PEAS framework can help translate users profiles into actionable findings in an easy and structured way, which is even more necessary when using behaviours extracted from users' telemetry data. In this way, raw (and potentially noisy) data can be transformed into concrete adaptation strategies through a process grounded in games and gamification literature. While assisting the decision-making process, the framework can also highlight aspects of the system that could be made adaptable to broaden the reached audience. Second, as we briefly showed in our analysis, using a structured tailoring approach can ease the tracking and update of adaptation strategies — i.e., dynamic adaptation - translating consistent changes of in-game behaviours directly to their effect on the adaptation strategy. Dynamic adaptation has been highlighted as an important future venue for gamification research [24]. Third, the PEAS framework enables the combination of different users profiles to derive the final adaptation strategy, which, alongside dynamic adaptation, has been found advantageous. Finally, relying on a shared adaptation framework would ease the replicability of the technique, especially in similar domains, as well as the systematic comparison of adaptation approaches.

\subsection{Limitations and Future Works}

The main limitation of our work is that the analysis was carried out on a single gamified app, which does not include all the factors of the PEAS framework. Nevertheless, the purpose was to show how the PEAS framework can be exploited in a gameful environment and does not need a 360-degree adaptation to be employable. Instead, the framework helps to decouple the adaptation aspect and clearly identify the possible adaptation strategies. However, researchers should explore further the usage of the PEAS framework in gamification, especially to compare adaption strategies across different applications or settings. Additionally, in this paper, we only showed how the PEAS framework could assist dynamic adaptations. Yet, this is an aspect that needs more in-depth and dedicated research to fully report the potential of a shared adaptation framework (i.e., PEAS). An additional limitation derives from the PEAS framework itself, as humans define the translation functions from users profiles towards the PEAS representation. This is, however, an issue recurrent in most works tackling adaptive gamification. While profiles can be built automatically, the conversions to actual intervention on the gameful app are defined by the domain experts. Therefore, future research should also investigate ways to automatize the process and attenuate humans' bias. Another limitation is related to the type of research, which is an uncontrolled study. Finally, data is collected without the possibility to identify the interference of external factors in the experience of the participants, such as weather and holidays. However, this issue is attenuated by environmental factors being shared by all the participants, as they are geographically located in the same area.

\section{Conclusions}

Adaptive gamification is becoming a notably superior approach to one-fits-all design strategies because of their many disadvantages. The complexity of user profiling-essential in the adaptation process-stems from a difficult decision-making process. Designers and analysts face many decisions, which can impact the outcome of a gamification strategy, and-in turn-the user experience. In this work, we showed that the PEAS framework can be used in gamification to translate user behaviours into actionable adaptation strategies. We then discuss how using this framework can foster replicability and comparability, and can assist dynamic adaptation.

\section{References}

[1] M. Busch, E. Mattheiss, R. Orji, A. Marczewski, W. Hochleitner, M. Lankes, L. E. Nacke, and M. Tscheligi, "Personalization in serious and persuasive games and gamified interactions," in Proceedings of the 2015 Annual Symposium on Computer-Human Interaction in Play, pp. 811-816, ACM.

[2] G. F. Tondello and L. E. Nacke, "Validation of user preferences and effects of personalized gamification on task performance," Frontiers in Computer Science, vol. 2, p. 29, 2020.

[3] S. C. Bakkes, P. H. Spronck, and G. van Lankveld, "Player behavioural modelling for video games," Entertainment Computing, vol. 3, no. 3, pp. 71-79, 2012.

[4] G. N. Yannakakis, P. Spronck, D. Loiacono, and 
E. André, "Player modeling," 2013.

[5] S. Hallifax, A. Serna, J.-C. Marty, and E. Lavoué, "Adaptive gamification in education: A literature review of current trends and developments," in European Conference on Technology Enhanced Learning, pp. 294-307, Springer, 2019.

[6] E. Lavoué, B. Monterrat, M. Desmarais, and S. George, "Adaptive gamification for learning environments," IEEE Transactions on Learning Technologies, vol. 12, no. 1, pp. 16-28, 2018.

[7] J. Pfau, J. D. Smeddinck, and R. Malaka, "Enemy within: Long-term motivation effects of deep player behavior models for dynamic difficulty adjustment," in Proceedings of CHI, pp. 1-10, 2020.

[8] M. Böckle, I. Micheel, M. Bick, and J. Novak, "A design framework for adaptive gamification applications," in Proceedings of HICSS 51, 2018.

[9] A. Knutas, R. Van Roy, T. Hynninen, M. Granato, J. Kasurinen, and J. Ikonen, "A process for designing algorithm-based personalized gamification," Multimedia Tools and Applications, vol. 78, no. 10, pp. 13593-13612, 2019.

[10] E. Loria, "A framework to infer player experience and inform customized content generation in gameful systems," in CHI PLAY Ext. Abstracts, pp. 21-28, 2019.

[11] S. Snodgrass, O. Mohaddesi, J. Hart, G. R. Rodriguez, C. Holmgård, and C. Harteveld, "Like peas in pods: the player, environment, agents, system framework for the personalization of digital systems," in Proceedings of FDG, pp. 1-15, 2019.

[12] S. Snodgrass, O. Mohaddesi, and C. Harteveld, "Towards a generalized player model through the peas framework,' in Proceedings of FDG, pp. 1-7, 2019.

[13] S. Deterding, D. Dixon, R. Khaled, and L. Nacke, "From Game Design Elements to Gamefulness: Defining Gamification," Proceedings of MindTrek, pp. 9-15, 2011.

[14] L. De-Marcos, E. Garcia-Lopez, and A. Garcia-Cabot, "On the effectiveness of game-like and social approaches in learning: Comparing educational gaming, gamification \& social networking," Computers and Education, vol. 95, pp. 99-113, 42016.

[15] B. Morschheuser and J. Hamari, "The Gamification of Work: Lessons From Crowdsourcing," Journal of Management Inquiry, vol. 28, pp. 145-148, 42019.

[16] J. Hamari and H. Sarsa, "Does Gamification Work? - A Literature Review of Empirical Studies on Gamification," HICSS, pp. 3025-3034, 2014.

[17] L. E. Nacke and S. Deterding, "The Maturing of Gamification Research," Computers in Human Behavior, vol. 71, pp. 450-454, 2017.

[18] J. Koivisto and J. Hamari, "The rise of motivational information systems: A review of gamification research," vol. 45, pp. 191-210, 42019.

[19] E. Marcucci, V. Gatta, and M. Le Pira, "Gamification design to foster stakeholder engagement and behavior change: An application to urban freight transport," Transportation Research Part A: Policy and Practice, vol. 118, pp. 119-132, 122018.

[20] J. Hamari, "Transforming homo economicus into homo ludens: A field experiment on gamification in a utilitarian peer-to-peer trading service," Electronic Commerce Research and Applications, vol. 12, pp. 236-245, 72013.
[21] R. M. Ryan, C. S. Rigby, and A. Przybylski, "The motivational pull of video games: A self-determination theory approach," Motivation and Emotion, vol. 30, pp. 347-363, 122006.

[22] E. L. Deci and R. M. Ryan, "The "what" and "why" of goal pursuits: Human needs and the self-determination of behavior," Psychological Inquiry, vol. 11, no. 4, pp. 227-268, 2000.

[23] R. Orji, R. L. Mandryk, and J. Vassileva, "Improving the efficacy of games for change using personalization models," ACM Transactions on Computer-Human Interaction, vol. 24, 102017.

[24] A. C. T. Klock, I. Gasparini, M. S. Pimenta, and J. Hamari, "Tailored Gamification: A Review of Literature," International Journal of Human Computer Studies, vol. 144, no. September 2019, 2020.

[25] R. Sifa, A. Drachen, and C. Bauckhage, "Profiling in games: Understanding behavior from telemetry," Social Interactions in Virtual Worlds: An Interdisciplinary Perspective, 2018.

[26] S. Makarovych, A. Canossa, J. Togelius, and A. Drachen, "Like a dna string: Sequence-based player profiling in tom clancy's the division," in Proceedings of AIIDE, York, 2018.

[27] A. Marczewski, "Even ninja monkeys like to play," CreateSpace Indep. Publish Platform, Charleston, Chapter User Types, pp. 69-84, 2015.

[28] S. Hallifax, E. Lavoué, and A. Serna, "To tailor or not to tailor gamification? an analysis of the impact of tailored game elements on learners' behaviours and motivation," in International Conference on Artificial Intelligence in Education, pp. 216-227, Springer, 2020.

[29] B. Barna and S. Fodor, "A data-driven approach to analyze user behavior on a personalized gamification platform," in International Conference on Games and Learning Alliance, pp. 266-275, Springer, 2019.

[30] E. Loria and A. Marconi, "Reading between the lines-towards an algorithm exploiting in-game behaviors to learn preferences in gameful systems," in Proceedings of FDG, pp. 1-12, 2020.

[31] W. Oliveira, A. Toda, P. Toledo, L. Shi, J. Vassileva, I. I. Bittencourt, and S. Isotani, "Does tailoring gamified educational systems matter? the impact on students' flow experience," 2020.

[32] L. Rodrigues, A. M. Toda, P. T. Palomino, W. Oliveira, and S. Isotani, "Personalized gamification: A literature review of outcomes, experiments, and approaches," in Proceedings of Technological Ecosystems for Enhancing Multiculturality, pp. 699-706, 2020.

[33] A. Marconi and E. Loria, "Sustainable Mobility in Smart Cities: The Key Role of Gamified Motivational Systems for Citizens' Engagement and Behavior Change," in Implications of Mobility as a Service (MaaS) in Urban and Rural Environments, pp. 211-246, IGI Global, 2020.

[34] A. Field, "Discovering statistics using spss, fourth edition," 2013.

[35] G. F. Tondello, K. Arrambide, G. Ribeiro, A. J.-1. Cen, and L. E. Nacke, ““ii don't fit into a single type": A trait model and scale of game playing preferences," in IFIP, pp. 375-395, Springer, 2019. 